\title{
Simulación de variables climáticas en ensayos de daño acelerado de pavimentos a escala natural
}

\section{Simulation of weather conditions on full scale accelerated pavement testing}

\section{Fabricio Leiva Villacorta}

National Center for Asphalt Technology,

Auburn University, AL, USA.

leivafa@auburn.edu

\section{Edgar Camacho Garita}

Laboratorio Nacional de Materiales y Modelos Estructurales de la Universidad de Costa Rica, San Pedro de Montes de Oca, San José, Costa Rica

edgar.camachogarita@ucr.ac.cr

\section{José Pablo Aguiar Moya}

Laboratorio Nacional de Materiales y Modelos Estructurales de la Universidad de Costa Rica, San Pedro de Montes de Oca, San José, Costa Rica

jose.aguiar@ucr.ac.cr

Fecha de recepción: 30 de mayo de 2016 / Fecha de aprobación: 18 de julio de 2016

\section{RESUMEN}

Los ensayos de daño acelerado de pavimentos a escala natural proveen mucha información acerca del comportamiento real de estructuras de pavimentos sujetas a solicitaciones mecánicas específicas. Si bien este es el principal factor que provoca daño en los pavimentos, el clima afecta también el desempeño de los mismos y no se ha estudiado ampliamente en ensayos a escala natural, ni mucho menos incluir su efecto de forma acelerada. En este artículo se describe parte de la implementación de un sistema para incluir de forma acelerada las variables climáticas en el laboratorio de pavimentos a escala natural PAVELAB del LanammeUCR. El sistema cuenta con capacidades únicas, entre las que se listan simulación de altas y bajas temperaturas, viento, lluvia y radiación solar. Se muestran algunos resultados de las primeras pruebas realizadas con el nuevo sistema y se analizan algunas de las posibles variedades climáticas de interés, para caracterizar el clima de Costa Rica en los ensayos que se realizan en el PAVELAB.

PALABRAS CLAVE: Ensayos acelerados, variables climáticas, envejecimiento, clima Costa Rica.

\section{ABSTRACT}

Full scale accelerated pavement testing provides much more information about the actual behavior of pavement structures subject to specific mechanical stresses. While this is the main factor causing damage to pavements, climate also affects performance and has not been extensively studied in full scale tests, much less its effect on an accelerated basis. This article describes part of the implementation of a system to include an accelerated form of weather variables in the laboratory full-scale pavement facility called PAVELAB of LanammeUCR. The system has unique capabilities including simulation of high and low temperatures, wind, rain and solar radiation. Some results of the first tests with the new system are shown in this paper and some of the possible climatic varieties of interest to characterize the climate of Costa Rica in the tests performed in the PAVELAB are also shown.

KEYWORDS: Accelerated testing, weather conditions, aging, Costa Rica. 


\section{INTRODUCCIÓN}

Los países que han mostrado un desarrollo significativo en el área de pavimentos lo han alcanzado, tradicionalmente, a través de la realización de ensayos en tiempo real (RTL, por sus siglas en inglés), debido a que esta técnica no requiere de grandes equipos especializados para la realización de los ensayos (LTPP, 2009 \& Metcalf, 1996). El tiempo necesario para llevar a cabo el ensayo (más de 10 años de monitoreo continuo de un tramo experimental) está asociado con una gran cantidad de complicaciones, ya que muchos de los tramos experimentales están ubicados sobre carreteras en operación.

En el caso de Costa Rica, se considera que, debido a la gran variabilidad climática, de materiales y tráfico a la que están sometidos los pavimentos, el costo de desarrollar un programa adecuado de ensayos RTL que abarque todas estas condiciones por períodos prolongados es prohibitivo. Sin embargo, existe la gran necesidad de poder caracterizar el desempeño a largo plazo de las estructuras de pavimentos del país como método único de poder desarrollar y calibrar metodologías de diseño. Para este propósito se considera que el uso de un equipo de Ensayo Acelerado de Pavimentos (APT en ingles) es idóneo.

Con el fin de poder contar en Costa Rica con pistas para ensayos APT, se realizó un estudio de diferentes equipos y se determinó que, para las condiciones tanto técnicas y económicas del país, el Simulador de Vehículos Pesados (Heavy Vehicle Simulator, HVS), es el equipo que mejor se adapta y cuyos resultados serán de mayor impacto en el corto, mediano y largo plazo para el país (Figura 1). Específicamente se ha determinado que el HVS es el equipo que se ajusta a los requerimientos de (Coetzee et al., 2008; Harvey et al., 1999 \& Leiva et al., 2013).

- Movilidad: Se trata de un equipo que se puede utilizar tanto dentro de instalaciones previamente acondicionadas o bien, puede ser fácilmente transportado a una sección de interés de una carretera.

- Evaluación acelerada de pavimentos: Es capaz de simular el deterioro de hasta 20 años que sufre una carretera entre 3 a 6 meses aproximadamente.

- Cargas reales: Puede controlarse la cantidad y la ubicación de las aplicaciones de carga.

- Compatibilidad: Los datos obtenidos entre otros equipos similares son compatibles entre sí.

- Desarrollo de modelos calibrados: Los resultados obtenidos permitirán mejorar y calibrar modelos de respuesta y desempeño, y asociarlos con los criterios de diseño y/o métodos de ensayo de laboratorio.
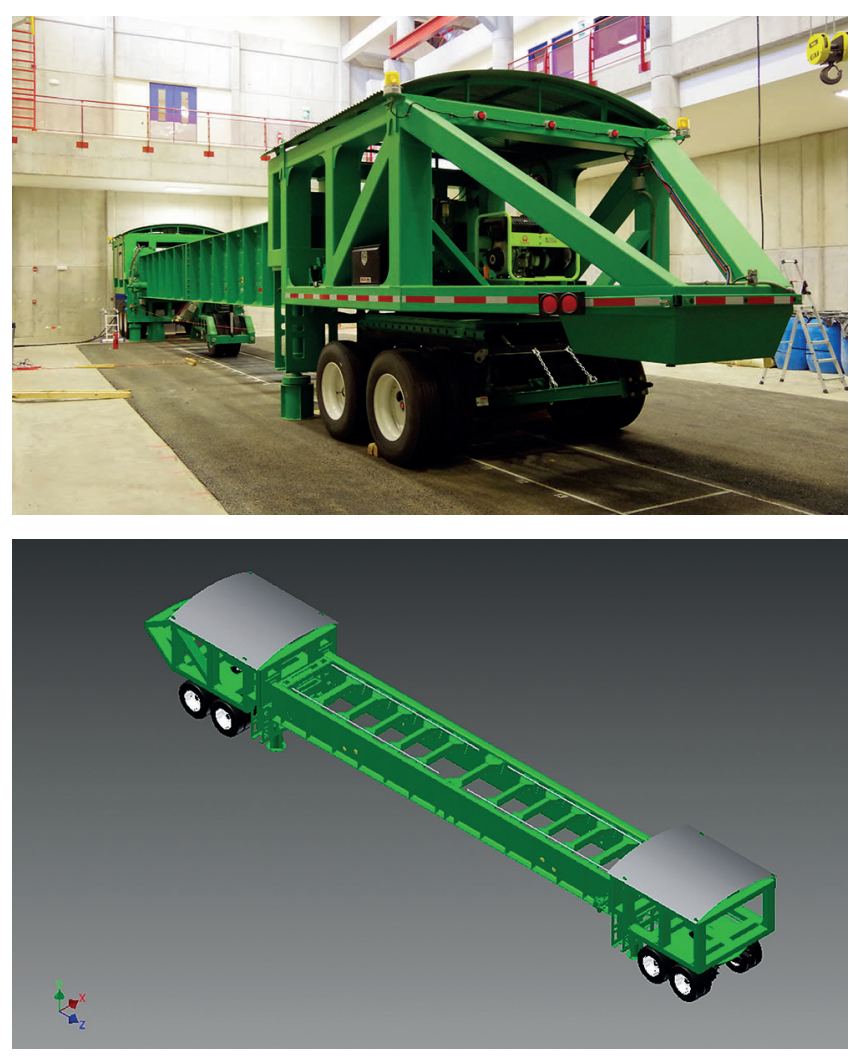

Figura 1. Equipo para ensayo de pavimentos a escala real del LanammeUCR,

HVS Mark VI.

\subsection{Productos Esperados}

El uso del HVS será fundamental en asegurar un gran avance en cuanto al nivel de investigación realizado por el LanammeUCR en el tema de ingeniería vial y por medio del cual se posibilitará la generación de una serie de productos tales como:

- Metodología de diseño mecanística - empírica de pavimentos, basándose en las condiciones de materiales, climáticas, de tráfico y constructivas reales de Costa Rica.

- Desarrollo de un software que permita la implementación de la metodología de diseño estructural de pavimentos para Costa Rica, que se base en los modelos de desempeño obtenidos mediante el uso de HVS.

- Desarrollo de nuevas especificaciones de materiales que se basen en el desempeño real y aporte estructural de los materiales en campo.

- Optimización de estructuras de pavimentos en uso en el país, basándose en las condiciones climáticas, estructurales, de materiales y tráfico de la zona específica donde se planea construir la estructura.

- Posibilidad de evaluación de materiales mejorados o nuevos materiales en una estructura de pavimento real. 


\section{EFECTO CLIMÁTICO EN PAVIMENTOS}

Un pavimento idealmente diseñado y construido debe ser capaz de resistir las variaciones estacionales del clima. Los cambios de temperatura, las fuertes lluvias, aunadas a malos drenajes y poco mantenimiento, exponen la infraestructura vial del país a deterioros acelerados. Para evitar una falla prematura de la estructura del pavimento, se debe considerar en el diseño todos los factores que afectan la variación de la temperatura del pavimento y el contenido de humedad de las capas granulares. La determinación de dicha variación requiere la utilización de modelos climáticos. Estos incluyen los modelos de transferencia de calor para determinar la distribución espacial y temporal de temperaturas, los modelos de equilibrio para determinar la distribución final de humedades a nivel de la subrasante y los modelos de infiltración y drenaje para poder predecir el grado de saturación en capas granulares.

Los modelos climáticos requieren los parámetros climáticos y las propiedades térmicas de los materiales. Los datos climáticos incluyen las temperaturas del aire promedio, máximas y mínimas, la velocidad del viento, la precipitación y la radiación. Las propiedades térmicas incluyen la conductividad térmica, el calor específico y el punto de fusión de los materiales. El clima y el tráfico son los factores externos más influyentes en el pavimento, pueden ocasionar un progresivo deterioro a lo largo de su vida útil. Los principales factores ambientales que influyen en el desempeño de la estructura se explican a continuación.

\subsection{Afectaciones de la precipitación}

El efecto de la humedad en el pavimento se determina según la precipitación, las condiciones geográficas, la saturación, la infiltración, las condiciones topográficas y las condiciones de drenaje. La cantidad y la intensidad de las precipitaciones, en forma de lluvia o nieve, afectan la profundidad del nivel freático y la cantidad del agua superficial que se infiltra en el subsuelo.
El agua puede ingresar a la estructura del pavimento por infiltración a través de grietas en la carpeta asfáltica, infiltración de agua a través de los espaldones, zanjas laterales o cunetas sin pavimentar, filtración desde un terreno más alto que la carretera, por fluctuaciones del nivel freático y por la ascensión capilar desde el nivel freático. En todos los casos estos aspectos contribuyen a la degradación de la capacidad soportante de la estructura.

El agua afecta la estructura del pavimento modificando o alterando las propiedades de los materiales como el módulo resiliente, la resistencia al esfuerzo cortante, la cohesión, la susceptibilidad a la expansión y contracción, la erosión, el grado de compactación, el envejecimiento del asfalto y la adherencia entre los agregados y el asfalto.

\subsubsection{Materiales granulares y suelos}

El módulo resiliente (Mr) es una propiedad empleada para evaluar la respuesta de un material, es un dato de entrada para el diseño, la evaluación y el análisis de pavimentos. El módulo resiliente se mide en ensayos de carga cíclica, y se define como el cociente entre el esfuerzo desviador aplicado y la deformación recuperable (elástica) medida. Este módulo se refiere a la capacidad de un material de recuperar su forma original después de haber sido deformado, por lo que no es indicador de la resistencia de un material sino una medida de la rigidez del material y un indicador de su calidad o condición. En la Tabla 1 se presentan los factores relacionados con la humedad y presión hidrostática que modifican el módulo resiliente de los materiales granulares y suelos, así como la manera en que los modifica.

En suelos de grano grueso el comportamiento ante la aplicación de las cargas rige por la fricción entre partículas y la reorientación de las partículas, el módulo resiliente en estos materiales incrementa con el incremento de los esfuerzos invariantes $\left(\sigma_{1}, \sigma_{2}, \sigma_{3}\right)$. El agua en este tipo de suelos tiende a escurrir rápidamente por su naturaleza

Tabla 1. Factores que afectan el módulo resiliente de los materiales granulares y el efecto de aumentar el valor del factor (Austroads Ltd . 2012)

\begin{tabular}{|c|c|}
\hline Factor & Efecto en el Mr de incrementar el factor \\
\hline Humedad de compactación & Crece hasta el valor de humedad óptimo y luego decrece \\
\hline Presión normal promedio & Crece en material granular y decrece en suelos \\
\hline Esfuerzo cortante & Decrece \\
\hline Contenido de humedad & Decrece \\
\hline
\end{tabular}


permeable y los vacíos entre partículas. En los suelos de grano fino el módulo resiliente decrece con el incremento del esfuerzo desviador dinámico $\left(\sigma_{\mathrm{d}}\right)$, este efecto se asocia al contenido de humedad del material. Cuando el suelo está saturado y se aplica una carga, la presión de poro aumenta y no se disipa rápidamente, esto ocasiona que el esfuerzo efectivo disminuya generando una reducción del módulo resiliente. Por su naturaleza cambiante, existe variaciones estacionales de módulos resilientes en este tipo de suelos. La reacción ante una alteración del contenido de humedad depende de la naturaleza del material. En general, se puede afirmar que (Austroads Ltd . 2012):

- Para suelos arenosos, pequeñas fluctuaciones en el contenido de humedad producen pocos cambios en el volumen y módulo resiliente.

- Para suelos limosos, pequeñas fluctuaciones en el contenido de humedad producen pocos cambios en el volumen, pero pueden producir grandes cambios en el módulo resiliente.

- Para los suelos arcillosos o suelos granulares con grandes contenidos de finos, pequeñas fluctuaciones en la humedad pueden producir grandes variaciones en el volumen (caso de arcillas expansivas) y disminuciones en el módulo del material.

\subsubsection{Afectaciones por los cambios de temperatura}

Las variaciones en la temperatura afectan principalmente la capa asfáltica del pavimento dado que las propiedades, el comportamiento y la estabilidad de la mezcla asfáltica varían de acuerdo a los cambios de temperatura. Materiales estabilizados con algún agente viscoelástico también son afectados por los cambios térmicos, como las bases estabilizadas con asfalto, así como las losas de concreto y las bases estabilizadas con cemento.

La relación entre la temperatura del aire y la temperatura del pavimento no es necesariamente lineal. Esto se debe a que el perfil de temperatura del material asfáltico requiere información de las propiedades térmicas del asfalto, las condiciones ambientales, el espesor de las capas, entre otros factores (Mena, 2013).

$\mathrm{Al}$ igual que en los materiales granulares, el módulo resiliente de la mezcla asfáltica es una propiedad que caracteriza de manera racional el comportamiento esfuerzo - deformación. Este módulo se deriva de la fricción entre las partículas de agregado, la viscosidad del ligante asfáltico, la cohesión de la mezcla y la adhesión entre el ligante y el agregado.
Las mezclas asfálticas son materiales viscoelásticos con propiedades que dependen de la temperatura y la frecuencia de carga. A bajas temperaturas predomina su comportamiento elástico, actúa como un sólido, se vuelve duro, quebradizo y su rigidez aumenta. A altas temperaturas el asfalto se vuelve menos elástico y más viscoso, y el módulo de la capa asfáltica se reduce significativamente.

A consecuencia de esta propiedad viscoelástica, el pavimento a altas temperaturas no puede soportar cargas vehiculares eficazmente como si puede a temperaturas más bajas. A altas temperaturas aumenta la componente plástica de las deformaciones del asfalto, por lo tanto, es propenso a sufrir deformaciones permanentes ante las cargas. A temperaturas bajas el asfalto es susceptible a agrietarse dada la rigidización de la capa.

Otra característica del asfalto es la capacidad de reacción con el oxígeno presente en el aire y el agua. La oxidación de la estructura produce un endurecimiento o rigidización del asfalto, perdiendo las propiedades de adherencia y ocasionándole una mayor fragilidad. La oxidación se produce naturalmente a medida que transcurre la vida útil de la estructura, pero se genera de manera más acelerada a altas temperaturas.

\subsection{Influencia de la radiación solar}

Para estudiar la cantidad de calor que entra y sale de la superficie del pavimento es necesario establecer las propiedades térmicas que definen el flujo de calor a través del pavimento, e identificar las condiciones del entorno. La temperatura del aire, velocidad del viento, y la radiación solar se utilizan para calcular el balance de calor en la superficie del pavimento (Johanneck, 2011). La transpiración, evaporación, condensación y sublimación tienden a omitirse en los cálculos de transferencia de calor porque sus cambios no afectan significativamente el balance de energía (NCHRP, 2004).

Los dos procesos por los que se suma o resta el calor en la superficie del pavimento son convección y radiación. La cantidad de radiación solar que incide sobre la superficie del pavimento depende de dos factores: la posición espacial del Sol, determinada por las coordenadas, el día y el año, y la cobertura de nubes. La fuente primaria del calor de radiación es la radiación solar de onda corta. La radiación realmente absorbida, del total que incide, depende de la capacidad de absorción de onda corta del pavimento, que está en función de la composición, color y textura superficial de la capa de rodadura. 
El otro tipo de flujo de calor inducido por la radiación es la radiación de onda larga. La radiación de onda larga es la radiación térmica que emite el pavimento de acuerdo con la teoría de la radiación del cuerpo negro, en la que dependiendo de la temperatura absoluta de un material una cantidad específica de calor es emitida. Una parte de esta radiación de onda larga, emitida por el pavimento y el ambiente que lo rodea, es reabsorbida por el pavimento luego de reflejarse en las nubes (NCHRP, 2004).

La radiación solar también es importante porque controla la evaporación del agua en la superficie del terreno, lo que induce un movimiento del agua en forma de vapor dentro de la estructura. Este movimiento es perjudicial porque favorece la separación del agregado y el asfalto.

\section{CONCEPTUALIZACIÓN DE UNA CÁMARA AMBIENTADORA}

Para el desarrollo de la cámara ambientadora que trabaje de acuerdo a las necesidades de los ensayos acelerados de pavimentos en el PAVELAB se enumeran los requerimientos básicos necesarios para su construcción:

- La cámara debía consistir en una estructura metálica modular con propiedades termo-acústicas, que se coloque sobre toda la sección central del HVS formando un volumen de control que permite aislar la pista de pruebas de su entorno.

- Esta estructura debía tener una estructura de techo similar a la del HVS.

- Dentro de la estructura se requería un sistema de lámparas, los cuales se encargarán de simular la radiación solar sobre la pista y aumentar la temperatura en el volumen de control.

- Para simular lluvia se planeó un sistema hidráulico de riego sobre la pista. Además, debía contar con todas las previstas necesarias para acoplarle un sistema de refrigeración eventualmente, si se determina que sea necesario simular bajas temperaturas con el HVS.

- Todos los sistemas serían manejados por un sistema centralizado de control, el cual debería permitir control en sitio a través de una pantalla y remoto a través de computadora.
La Figura 2 muestra un diagrama conceptual de la estructura de la cámara que rodea al equipo HVS. Los accesos al interior del equipo son de suma importancia para la adquisición de datos de sensores embebidos y levantamiento de daños estructurales del pavimento.

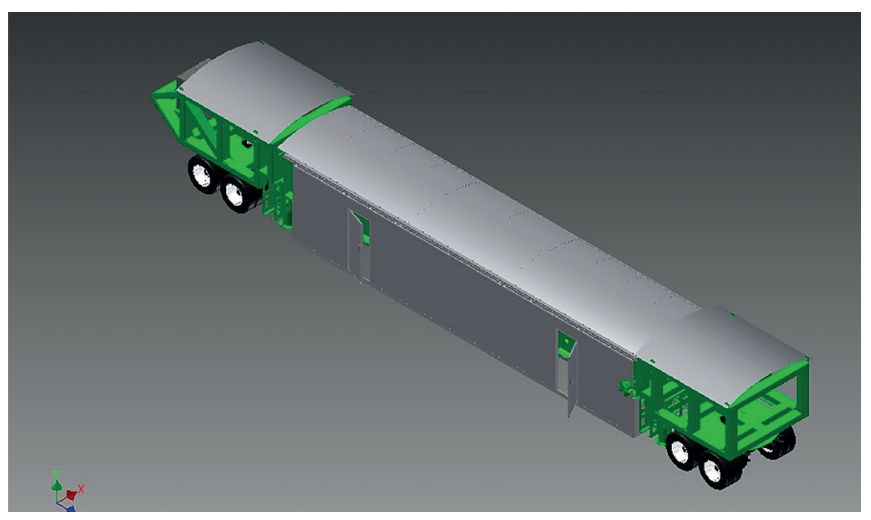

Figura 2. Conceptualización de la cámara ambientadora

\section{CONSTRUCCIÓN DE LA CÁMARA AMBIENTADORA}

La cámara de acondicionamiento (Figura 3) para los tramos y ensayos a realizar con el simulador de vehículos pesados (HVS) consiste finalmente en una serie de paneles que junto al equipo definen un espacio cerrado de condiciones ambientales (controladas y regulables) sobre el tramo en estudio. Dicho conjunto de paneles tiene la capacidad de aislar el calor interno, similar a un horno, y mantener una temperatura la cual se pueda ajustar. Considerando que se trata de un espacio amplio, se tomaron medidas de diseño para poder mantener la temperatura de forma uniforme en todo el tramo de ensayo. Otra característica que poseen los paneles es la instalación de dispositivos de luz ultravioleta, para envejecer la superficie de ensayo.

Así mismo la cámara tiene la posibilidad de lanzar agua de forma controlada sobre el tramo de estudio. Para esto se cuenta con un sistema con aspersores en el interior de la cámara que distribuyen de forma uniforme el agua.

Los paneles están formados por diferentes capas, que permitan estabilidad térmica y la resistencia al contacto con el agua. Finalmente, los paneles se pueden retirar de una manera simple, idealmente por una sola persona o dos personas y se ha dejado previsto para instalación a futuro el sistema de enfriamiento para desarrollar ensayos a temperaturas intermedias y bajas. 


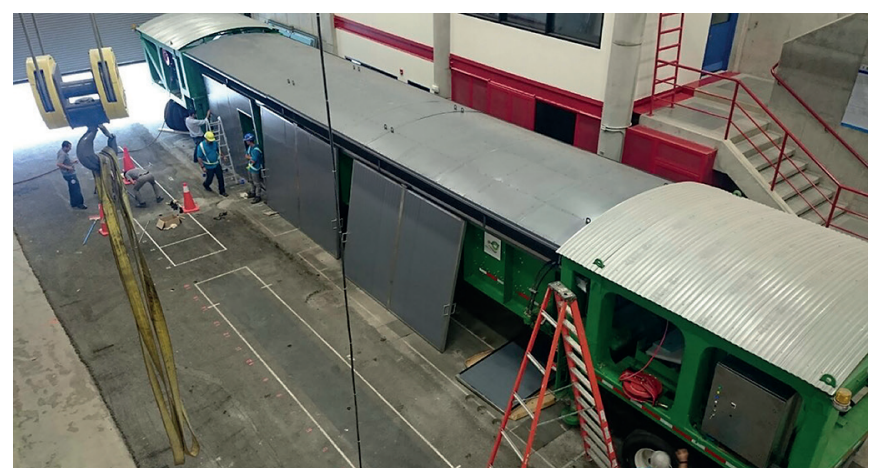

Figura 3. Construcción de la cámara ambientadora

\section{RESULTADOS PRELIMINARES}

En la Figura se presenta el perfil de temperaturas, la radiación solar y la precipitación para un día caluroso, y en la Figura 5 para un día frío en la ciudad de San José, Costa Rica. En un día caliente se lleva aproximadamente 6 horas para alcanzar la temperatura máxima del pavimento. En el día frío, la temperatura superficial del pavimento es muy similar a la temperatura del aire. Este tipo de información sirvió de base para el ensayo preliminar de calentamiento de la estructura de pavimento por medio de radiación lumínica dentro de un volumen de aire conocido.

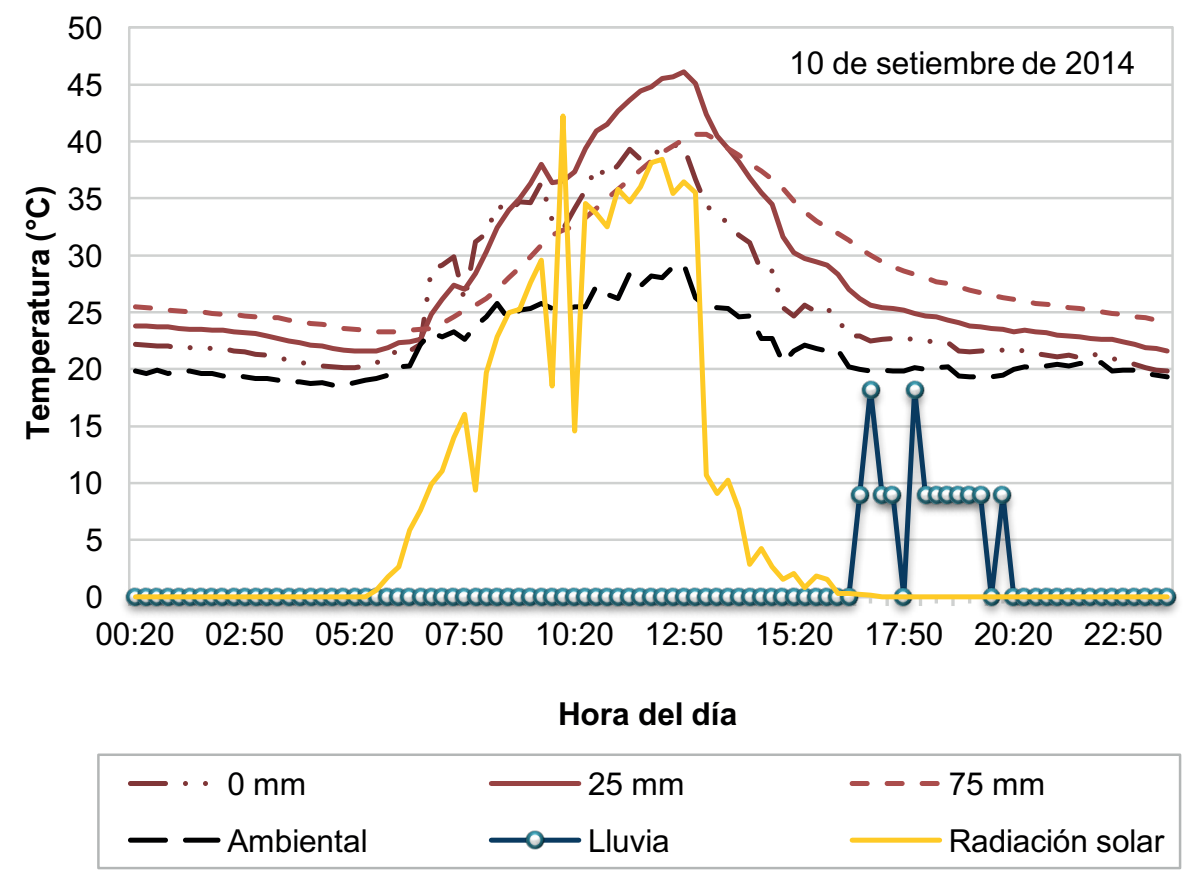

Figura 4. Perfil de temperatura del pavimento para un día caluroso (Perera, 2015).

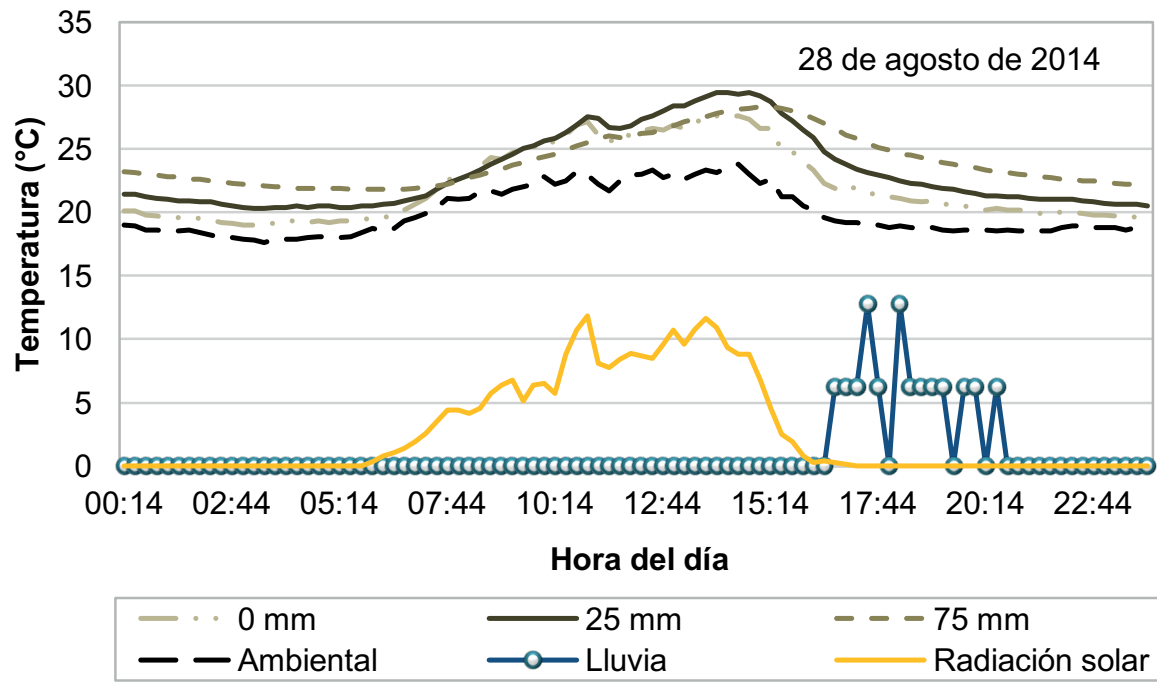

Figura 5. Perfil de temperatura del pavimento para un día frío (Perera, 2015). 
Las siguientes figuras muestran resultados preliminares llevados a cabo para la selección del sistema de calentamiento de la estructura de pavimento. La idea de este experimento fue el buscar el mecanismo más eficiente para acelerar el proceso de calentamiento natural observado en las Figuras 4 y 5. Para este caso se seleccionó un sistema de lámparas enfocadas hacia la superficie del pavimento de tal forma que no dañen los componentes eléctricos y cables del HVS. En una primera instancia se realizó el ejercicio de calentamiento mediante mecanismos de lámparas infrarrojas para observar el cambio de temperatura a nivel ambiental dentro de la cámara, a nivel externo de la cámara que se denominó como "Aire", luego se colocaron sensores en la estructura de pavimento a nivel de superficie, a $6 \mathrm{~cm}$ de profundidad, a $12 \mathrm{~cm}$ y a $15 \mathrm{~cm}$ de profundidad para cuantificar el gradiente térmico. La estructura evaluada cuenta con $12 \mathrm{~cm}$ de espesor de mezcla asfáltica y $24 \mathrm{~cm}$ de base estabilizada con cemento por lo que el sensor a mayor profundidad se encuentra $3 \mathrm{~cm}$ dentro de la base.
Como se observa en la Figura 6 el calentamiento con energía infrarroja proporcionó temperaturas superficiales cercanas a los $50^{\circ} \mathrm{C}$ en un poco más de 2 horas. Por otro lado, la temperatura dentro de la cámara, al igual que dentro del pavimento, se mantuvo por debajo de $35^{\circ} \mathrm{C}$. Y finalmente, el resto de la estructura sufrió un leve incremento de temperatura.

El segundo caso contempló el uso de energía provista por lámparas de halógeno que no resultaron tan eficientes como la energía infrarroja (Figura 7). Sin embargo, se utilizaron para simular la influencia de la radiación solar.

Finalmente, una combinación de ambas tecnologías permitió obtener temperaturas en el pavimento superiores y más cercanas a las que se utilizarían en el ensayo acelerado de pavimentos. De igual forma, al ser una energía enfocada al pavimento, la temperatura de la cámara y por lo tanto los componentes eléctricos del HVS no se verían tan afectados por el cambio término (Figura 8).

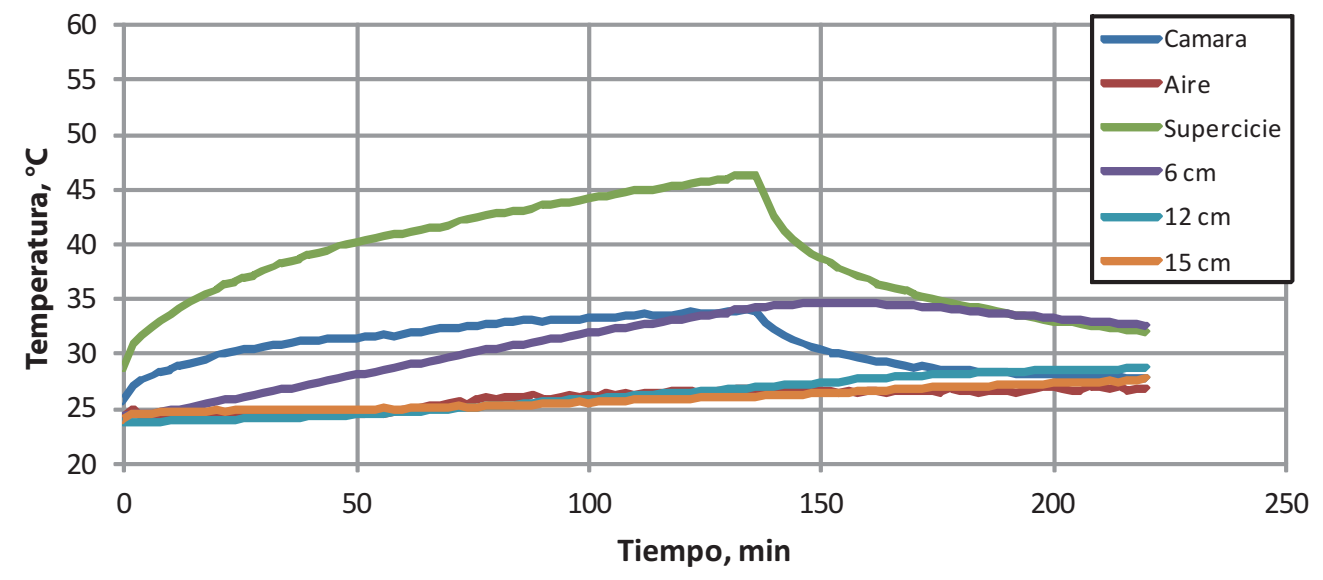

Figura 6. Calentamiento vía energía infrarroja

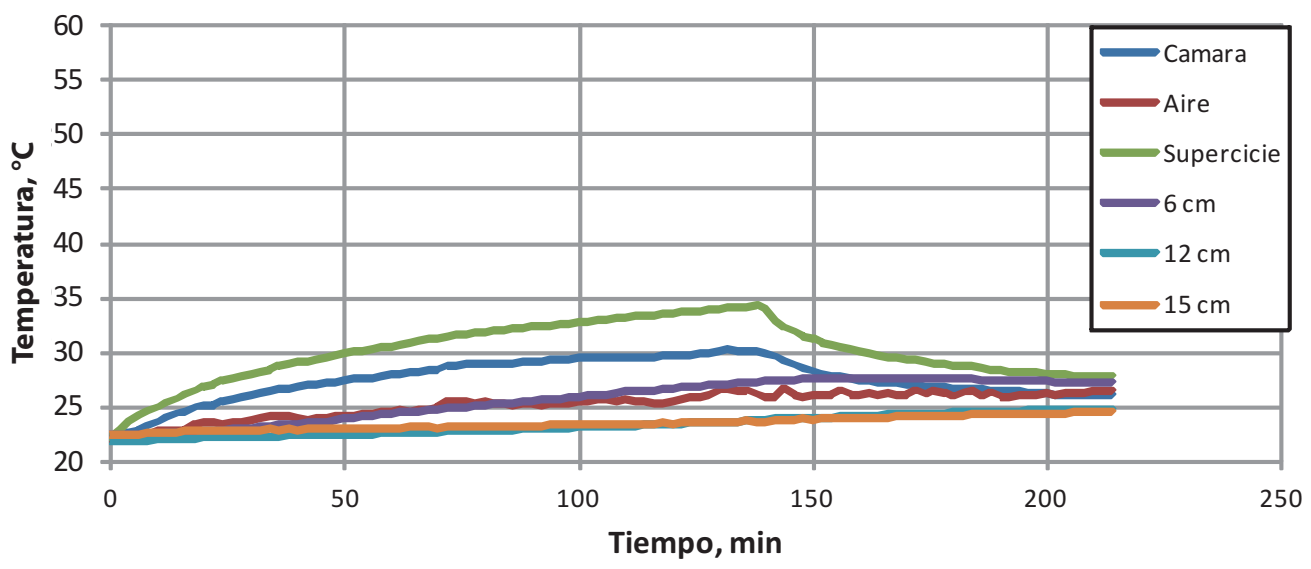

Figura 7. Calentamiento vía energía halógeno 


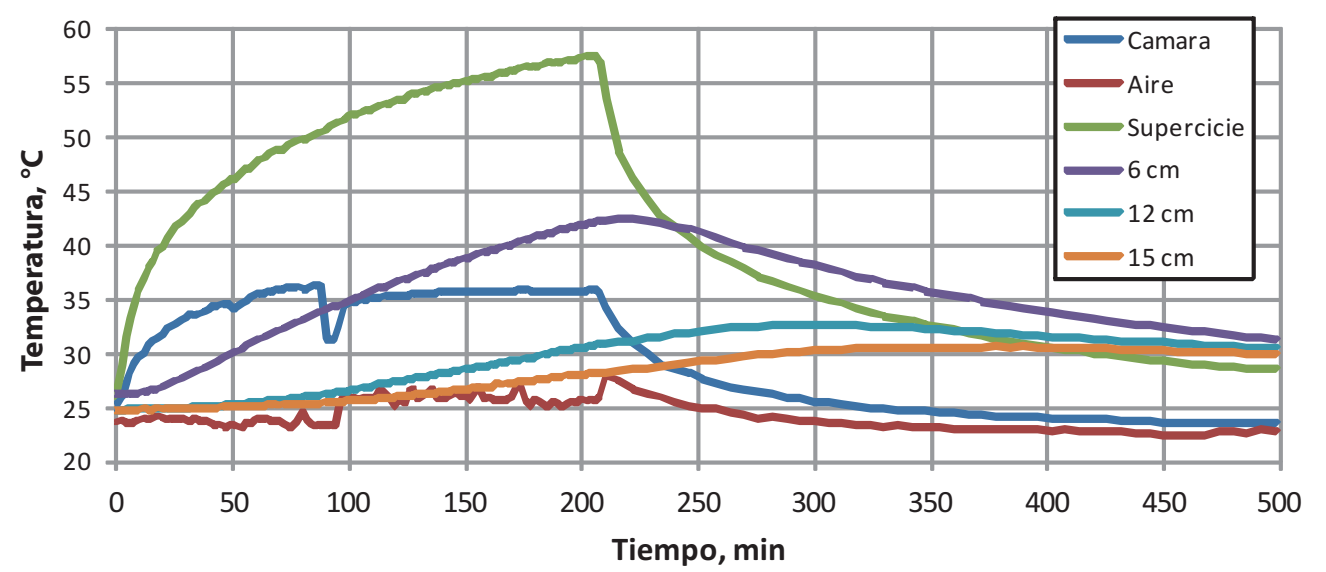

Figura 8. Calentamiento vía energía infrarrojo + halógeno

\section{CUANTIFICACIÓN DE DAÑO CAUSADO POR EL AGUA}

El criterio de incorporar humedad superficial a un tramo de ensayo, resulta de un proceso de ensayo caracterizado por la deformación de capa de ruedo, presencia de grietas leves sin que estas lleguen a interconectase y de difícil percepción a la vista. Una vez cumplido el criterio por deformación de la superficie $(900.000$ repeticiones) y con tiempo disponible en el programa de ensayos, se decide aprovechar la condición drenada de la estructura y someterla a humedad únicamente de forma superficial por 100.000 repeticiones de carga para cuantificar el daño causado por la presencia de agua superficial.

El principal efecto observado en la estructura, debido a la presencia de agua superficial, fue el movimiento de material fino proveniente de la capa base (bombeo de finos) a través de las grietas que se formaron en la capa de ruedo. Esta pérdida de material en la capa base generó un aumento en la deformación observada en la superficie, así como su agrietamiento (Figura 9).
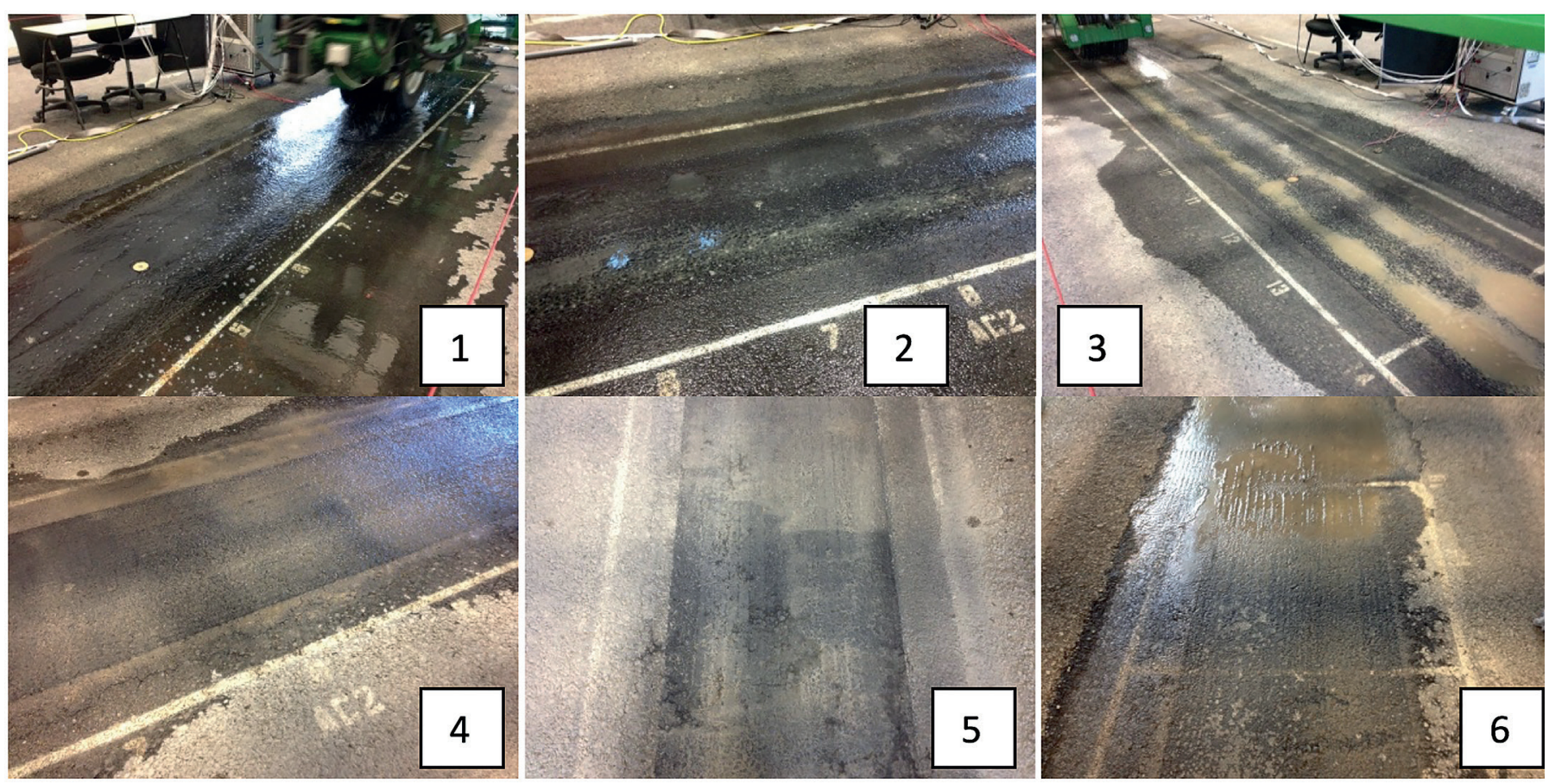

Figura 9. Evolución del tramo de ensayo 
En la secuencia de imágenes mostrada se puede apreciar la evolución de los deterioros presentados, el bombeo de material de la capa subyacente. Este material salió por las grietas que se formaron en la capa superficial durante la etapa seca del ensayo (900 mil repeticiones de carga), así como las grietas que se formaron posteriormente con la aplicación de cargas y agua (100 mil repeticiones adicionales).

\subsection{Factores que influyen en el deterioro presentado}

Este tipo de deterioros con bombeo de finos se pueden observar en Costa Rica cuando se colocan capas delgadas de mezcla asfáltica sobre bases granulares con malas condiciones de drenaje, así como cuando se utiliza una capa de base estabilizada con cemento, pero la misma presenta deficiencias constructivas.

El ingreso de la humedad y el respectivo bombeo de finos podrían limitarse mediante una oportuna colocación de un sello asfáltico de las grietas que también impermeabilice la superficie; retrasando también la deformación por el movimiento de la base granular.
Una fuente importante de información es la recolectada por medio de sensores laser incorporados al equipo HVS, los cuales miden la distancia del sensor a la superficie, de manera que la forma mediante una secuencia programada se recolecta una secuencia de líneas sobre la superficie, formando una malla de datos que representa de buena forma la superficie del tramo ensayado. Esta secuencia se recolecta aproximadamente cada 20.000 repeticiones de carga. La regularidad de la superficie, mediante el Índice de Regularidad Internacional se ha constituido en una importante referencia para poder asociar el desempeño general de las estructuras de pavimento y el servicio que se brinda a los usuarios.

En la Figura 10 se pueden apreciar los valores de regularidad calculados para los diferentes valores de elevaciones de superficie recolectados. De dicho gráfico resaltan los valores calculados para la zona ensayada, siendo estos los más representativos para el tramo. Se incluyen valores máximos y mínimos como parámetros de referencia; así como un cálculo de regularidad para la totalidad de los valores medidos, incluyendo en esto los valores fuera de la influencia directa del paso de la rueda.

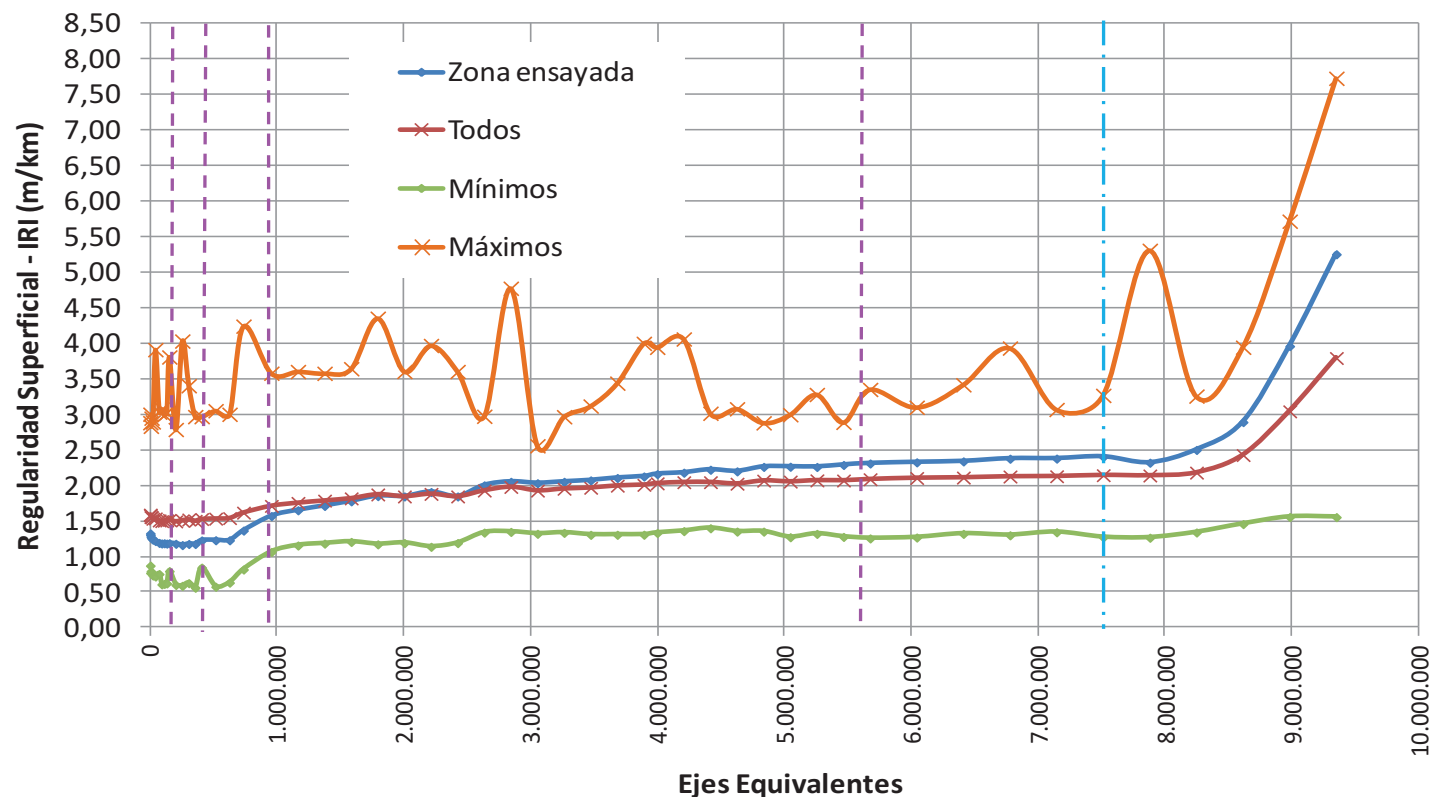

Figura 10. Variación de la regularidad superficial (IRI) a lo largo del tramo de ensayo (valores promedio, máximos y mínimos) 


\section{CONCLUSIONES Y FUTURO DEL ENSAYO ACELERADO}

De los resultados del estudio realizado se pueden obtener las siguientes conclusiones:

- Se vuelve sumamente necesario considerar el efecto climático al cual están expuestas las estructuras de pavimento. En especial en ensayos acelerados de pavimentos ya que dado el tiempo reducido de aplicación de carga puede dejar factores de interacción carga clima que son fundamentales para una cuantificación adecuada del desempeño.

- El diseño, construcción y funcionamiento de una cámara climática para ensayos acelerados a escala natural involucra un estudio detallado de las condiciones de campo que se pretenden reproducir. Adicionalmente, el funcionamiento de la cámara no debe interferir o afectar negativamente el funcionamiento del equipo de ensayos acelerados.
- En el caso de Costa Rica, el daño acelerado por la presencia de agua superficial es cuantificable a través del levantamiento de la regularidad superficial del pavimento que indiquen un estado y comportamiento de falla de los materiales.

- Este tipo de daño es más comúnmente apreciable en carreteras con alta precipitación por lo que se recomienda este tipo de ensayo con agua superficial aplicada de forma cíclica el cual acelere y duplique el efecto climático observado en campo.

- La siguiente etapa corresponderá a la saturación y aplicación de ciclos de calentamiento, envejecimiento con rayos ultravioleta y aplicación de agua superficial para un ensayo más completo de las estructuras.

- Dependiendo de la región, se vuelve necesario el uso de una cámara climática para controlar los cambios ambientales y para cuantificar un tipo de deterioro en específico, de no ser este el caso se vuelve difícil discernir los parámetros que propician un tipo de daño específico.

\section{REFERENCIAS}

1. Austroads Ltd. (2012). Guide to Pavement Technology Part 2: Pavement Structural Design. Recuperado el 4 de Febrero de 2014, de Austroads Publications Online.

2. Coetzee, $\mathrm{N}$ et al. (2008) The Heavy Vehicle Simulator in Accelerated Pavement Testing: Historical Overview and New Developments. 3rd International Conference APT.

3. Harvey, J. T., Hoover, T., Coetzee, N. F., Nokes, W. A., and Rust, F. C. (1999) Caltrans Accelerated Pavement Test (CAL/APT) Program-Test Results: 1994-1997. AAPT Symposiumon Accelerated Pavement Testing, Boston, MA, March 16-18.

4. https://www.onlinepublications.austroads.com.au/items/AGPT02-12.

5. Johanneck, L. A. (2011). A comprehensive evaluation of the effects of climate in mepdg predictions and of mepdg eicm model using mnroad data. Tesis para optar por el grado de maestría, University of Minnesota.

6. Leiva, Aguiar y Loría. (2013) Ensayos Acelerados De Pavimentos En Costa Rica. Revista Infraestructura Vial, Vol 15 (\#26), $33-$ 41. San José Costa Rica.

7. LTPP (2009). LTPP Beyond FY 2009: What Needs to Be Done?. Reporte FHWA-HRT-09-052.

8. Mena, W. (2013). Implementación del Modelo Climático de la MEPDG "AASHTO 2008" en Colombia para tres condiciones climáticas. Tesis de Maestría en Ingeniería, Universidad EAFIT, Escuela de Ingeniería, Medellín. Recuperado el 12 de Marzo de 2014, de http://repository.eafit.edu.co

9. Metcalf, J. B. (1996) NCHRP Synthesis of Highway Practice 235.

10. National Cooperative Highway Research Program. (2004). Guide for Mechanistic- Empirical Design of new and rehabilitated pavement structures. NCHRP 1-37A, TRB, National Research Council, Washington, DC.

11. Perera Lizano, Ariana. Proyecto final de graduación. (2015) Determinación Y Análisis De Los Factores Climáticos Críticos Para El Diseño De Pavimentos Flexibles En Costa Rica. Universidad de Costa Rica. 